\title{
Entre la letra y los niños: mediación del desarrollo para la literacidad
}

\section{Between letters and children: mediating literacy development}

DOI: https://doi.org/10.32870/dse.v0i23.744

\author{
Luis Felipe Gómez López* \\ Ma. Guadalupe Valdés Dávila** \\ Ma. de Lourdes Centeno Partida***
}

\begin{abstract}
Resumen
El artículo analiza las intervenciones mediacionales utilizadas en el desarrollo de la lectoescritura inicial en un grupo de ocho niños que presentan dificultades para su adquisición; pertenecen a familias de bajos recursos económicos y habitan en colonias del sur de la Zona Metropolitana de Guadalajara. El método utilizado fue un estudio cualitativo de caso. El objeto de análisis fueron las sesiones de clase realizadas durante el primer semestre de 2019 en un centro de atención comunitaria. Para el análisis y la interpretación se utilizó el marco teórico del constructivismo sociocultural. Los datos recabados permitieron identificar 12 acciones mediacionales agrupadas en tres grandes categorías: el sentido de la actividad, la enseñanza directa y el desarrollo de procesos reflexivos. Se concluye que las acciones mediacionales ayudaron al aprendizaje de los estudiantes, pero hizo falta un mayor seguimiento individual de cada uno para lograr mejores resultados.
\end{abstract}

Palabras clave: lectoescritura - literacidad - acciones mediacionales - dislexia.

\begin{abstract}
This article analyzes the mediating interventions used in the development of early literacy with a group of nine children who present difficulties in its acquisition. They belong to low-income families that live in the south of the Guadalajara Metropolitan Area. The study was a qualitative case analysis of the work sessions held in a community care center during the first semester of 2019. For the analysis and interpretation, the sociocultural constructivism theoretical framework was used. The data collected allowed us to identify twelve mediational actions grouped into three broad categories: the meaning of the activity, direct teach-
\end{abstract}

* Doctor en Educación. Profesor-investigador. SNI nivel I. Línea de investigación: currículo y modelos educativos, específicamente en el estudio de los procesos de enseñanza y aprendizaje. Departamento de Psicología, Educación y Salud del Instituto Tecnológico y de Estudios Superiores de Occidente (ITESO). México. Igomez@iteso.mx

** Doctora en Educación. Profesora-investigadora. Línea de investigación: aprendizaje, currículo, innovación y la formación docente. Departamento de Psicología, Educación y Salud del Instituto Tecnológico y de Estudios Superiores de Occidente (ITESO). México. pitina@iteso.mx

*** Maestra en Educación y Procesos Cognoscitivos. Profesora-investigadora. Línea de investigación: estudio de los procesos de enseñanza y aprendizaje, particularmente en la literacidad. Departamento de Psicología, Educación y Salud del Tecnológico y de Estudios Superiores de Occidente (ITESO). México. Icenteno@iteso.mx 
ing and the development of reflexive processes. We concluded that mediation processes helped children to learn, but more individual monitoring of each child's learning is required to achieve better results.

Keywords: reading and writing - literacy - mediational action - dyslexia.

\section{Introducción}

En el mundo hay 750 millones de jóvenes y adultos que no aprendieron a leer y escribir, además de 50 millones de niños que no tienen competencias básicas de lectura, escritura y cálculo (UNESCO, 2017). Este es un grave problema que impide a las personas integrarse plenamente a un mundo donde la comunicación mediante la letra impresa, en medios físicos y digitales, es fundamental.

La UNESCO (2017) considera que las competencias de lectoescritura son una parte intrínseca del derecho a la educación, ya que empoderan a las personas y comunidades y les permiten actuar plenamente en la sociedad; además, facilitan su participación en el mercado laboral, ayudan a que mejore la salud y la alimentación en las familias, así como a la reducción de la pobreza y la ampliación de las oportunidades de los individuos para desarrollarse a lo largo de la vida.

En México el problema de la falta de comprensión lectora en los estudiantes ha sido documentado por mucho tiempo. Desde 2007, 25\% de los niños de tercer grado de primaria estaban por debajo del nivel básico en comprensión lectora y reflexión sobre la lengua; 56\% apenas en el básico; $17 \%$ en el medio y solo $2 \%$ se encontraba en un nivel avanzado. Si se desagregan los niños indígenas de este mismo grado, $41 \%$ de ellos no alcanza el nivel básico (INEE, 2007). Mediciones más recientes muestran que el problema persiste. En 2018, 49\% de los alumnos de sexto grado evaluados con la prueba Planea se ubicaron en el nivel insuficiente. Solo $17.9 \%$ alcanzaron los niveles 3 y 4, satisfactorio y sobresaliente, respectivamente (INEE, 2019).

Esta dificultad permanece en otros niveles educativos. El más documentado ha sido el de $3^{\circ}$ de secundaria, que evalúa periódicamente la Organización para la Cooperación y el DesarroIlo Económico, (OCDE) mediante la prueba PISA, pero eso no significa que la dificultad lectora inicie en ese nivel sino que viene desde la primaria, pero en la secundaria no han logrado una mejora sustantiva.

En México, el puntaje promedio de 420 que obtuvieron los estudiantes de $3^{\circ}$ de secundaria en la prueba PISA 2018, es inferior al de 487, que fue el promedio de los demás países de la OCDE. Solo $1 \%$ alcanzó los niveles superiores de lectura, que en esa prueba son el 5 y el 6 . En contraste, $10 \%$ de los estudiantes de los países de la mencionada organización alcanzó ese nivel (OCDE, 2019). El nivel superior de lectura significa que los estudiantes pueden comprender textos largos que incluyen conceptos abstractos y son capaces de distinguir hechos de opiniones.

$55 \%$ de los estudiantes de secundaria de México, consiguió el nivel 2 de competencia lectora, lo que significa que pueden leer textos de longitud moderada e identificar en ellos la idea 
principal. Además, consiguen encontrar información cuando los criterios son explícitos (OCDE, 2019). Los bajos puntajes obtenidos por los estudiantes mexicanos no indican que todos tengan una competencia lectora poco sofisticada, pues hay variaciones importantes entre grupos y personas.

En el desarrollo inicial de la lectoescritura, de diversos países se ha informado tanto sobre problemas de enseñanza como en el desarrollo. Un ejemplo de esto se muestra en el estudio de Medina, Valdivia y San Martín (2014), quienes encontraron que en la enseñanza de la lectura inicial en Chile los profesores promovían interacciones en que el discurso dominante era el suyo, con escasa participación de los estudiantes y poca posibilidad de que estos adquirieran nuevo vocabulario y conceptos metalingüísticos, así como poco desafío para los lectores. Los docentes mejor evaluados tendían a plantear desafíos cognitivos a sus alumnos.

En cuanto a las dificultades, la más importante para el desarrollo de la lectoescritura es la dislexia. Esta presenta distintos niveles de prevalencia en los estudiantes dependiendo del instrumento de evaluación que se utilice, así como de la lengua y la cultura de estos, pero la mayoría la ubica entre 15 y 17\% de la población de niños en edad escolar (Tamayo, 2017; Fletcher et al., 2009; APA, 2014).

En el estudio del dominio de la lectoescritura hay una tendencia a denominarla literacidad, para desligarla de la idea común de que la lectura es la simple decodificación de un texto escrito y la escritura el acto de traducir los fonemas en grafías. Con el nuevo término se enfatiza "una amplia gama de competencias cognitivas, desde la decodificación básica, al conocimiento de palabras, gramática y estructuras y patrones textuales y lingüísticos más amplios hasta el conocimiento acerca del mundo" (OCDE, 2018: 28).

Durante la reunión internacional de expertos en 2003, la UNESCO definió la literacidad como: "la habilidad de identificar, comprender, interpretar, crear, comunicar y calcular, utilizando materiales escritos e impresos, asociados con contextos variados" (UNESCO, 2004: 13), pero la noción se ha ido ampliando hasta incluir la habilidad para utilizar el lenguaje, los números, las imágenes y las computadoras, así como para comprender y comunicarse utilizando los variados sistemas de símbolos de una cultura (UNESCO, 2006). También se han incluido "los roles que asumen el lector y el autor, los valores sociales asociados con las prácticas discursivas correspondientes, las formas de pensamiento que se han desarrollado con ellas" (Cassany, 2005: 1), entre otras. En palabras de Cassany (2006: 38), la literacidad "abarca todo lo relacionado con el uso del alfabeto: desde la correspondencia entre sonido y letra hasta las capacidades de razonamiento asociadas a la escritura".

Diversas definiciones de literacidad enfatizan los mismos aspectos centrales: el uso del lenguaje escrito, pero más allá de la mera decodificación. Massaro (2012: 324) la define como "la habilidad para utilizar el lenguaje escrito para funcionar bien en una cultura letrada, para perseguir metas independientes y adquirir el conocimiento requerido para una vida exitosa". El míni- 
mo necesario es leer fluidamente y comprender. La OCDE considera que la literacidad consiste en "comprender, usar, involucrarse con los textos escritos y reflexionar sobre ellos, para alcanzar metas propias, desarrollar el propio conocimiento y potencial y participar en la sociedad (OCDE, 2018: 27). Para este organismo internacional la literacidad tiene ciertos componentes que pueden variar según la lengua de que se trate, pero los que generalmente se reconocen, independientemente de la familia lingüística, son: significado de las palabras (vocabulario impreso), procesamiento de oraciones y comprensión de pasajes (OCDE, 2018: 27).

Gutiérrez y Díez (2017) señalan que los niños que no tienen dificultades, aprenden a leer con cualquier método que se les enseñe y que la mayoría se beneficia de que se trabaje de manera importante el desarrollo de habilidades fonológicas, sobre todo quienes presentan dislexia (Shaywitz, 2003). De acuerdo con esta última autora, las investigaciones de los últimos 30 años muestran que la dislexia, un trastorno caracterizado por "dificultades en el reconocimiento de palabras en forma precisa o fluida, deletrear mal y poca capacidad ortográfica" (Asociación Americana de Psiquiatría, 2014: 67), tiene como causa principal una dificultad en el módulo fonológico del cerebro, por lo que resulta fundamental que los niños desarrollen la habilidad de segmentar las palabras en fonemas y comprender el principio alfabético, es decir, que las grafías se relacionan con los fonemas, en número y en secuencia (2014: 44). Otros autores, como Moura, Pereira y Moreno (2020), también reconocen el déficit fonológico como causa de la disléxica, pero señalan que el déficit en la función nominativa del lenguaje es otra y que solo en algunos casos en ambos, a lo que refieren como el doble déficit.

La presente investigación utiliza el enfoque constructivista sociocultural, perspectiva que ubica al alumno como el centro del proceso de aprendizaje y constructor de su propio conocimiento. Para Vigotsky (1979), el aprendizaje es principalmente de naturaleza social, aunque puede haber ciertos procesos mentales inferiores que se desarrollan a partir del contacto del individuo con el mundo. Así, lo importante para la construcción de la literacidad son los procesos mentales superiores que se generan únicamente a través de la interacción social, por ello, a esta se le considera como un factor clave del aprendizaje.

Vigotsky (1979) plantea una relación triangular del aprendizaje en la que, además del sujeto que conoce y el objeto de conocimiento, se incluirían los instrumentos socioculturales que ayudarán al sujeto en la aprehensión de dicho objeto. Cuando el alumno aprehende un contenido utiliza los artefactos creados por la cultura: herramientas y símbolos (Vigotsky, 1979). Por eso se dice que los procesos psicológicos superiores están mediados por estos.

Desde esta perspectiva teórica, un concepto fundamental para el aprendizaje es el de zona del desarrollo próximo, propuesto por Vigotsky (1979), quien lo definía como aquella zona en la que el alumno no es capaz de realizar una actividad por sí mismo, pero puede hacerla con la ayuda de otra persona más competente. Para este autor, no tiene sentido ayudar al alumno en lo que él puede aprender por sí mismo, ni insistir en que aprenda aquello para lo que aún no 
está preparado. El aprendizaje solo ocurre en la zona en que el individuo puede desempeñar una actividad con la ayuda de otro.

La educación formal, en esta perspectiva, debería ayudar al alumno a desarrollar sus funciones psicológicas superiores y, con ello, el uso funcional, reflexivo, contextualizado y autorregulado de los instrumentos culturales, psicológicos y físicos, así como de aquellos contenidos que una cultura en particular considera valiosos (Baquero, 1996), en este caso, el código escrito y las distintas estrategias que permiten utilizarlo para propósitos comunicativos.

Para ayudar a los niños a generar el proceso psicológico de la literacidad, se utilizan ayudas a través de la zona del desarrollo próximo que se dan mediante lo que Bruner (Wood, Bruner y Ross, 1976) Ilamó "andamiaje", el cual consiste en un sistema de ayudas temporales que permiten que el aprendiz funcione en un nivel superior de pensamiento que el que tendría trabajando sin la ayuda de la persona más competente que le proporciona el "andamio". El andamiaje tiene tres características esenciales: debe ser ajustable a las necesidades del alumno participante, es temporal y es explícito (Wood, Bruner, Ross, 1976). Los andamios se presentan al alumno cuando hay contenidos muy específicos que habrán de aprenderse y que estos no pueden lograrlo solos, pero sí con la ayuda de los andamios que proporcionan los profesores u otros compañeros.

En el ámbito educativo, otro término más utilizado que el de andamiaje es el de mediación, que refiere al papel que juega un individuo al ubicarse ante el objeto de aprendizaje y el sujeto que aprende (Feuerstein, 1980). Haywood, Brooks y Burns (1991) enumeran siete características de la mediación eficaz: intencionalidad, trascendencia, comunicación de significado y propósito, mediación del sentimiento de ser competente, regulación de conducta y participación compartida.

Con esas características se refieren a que el mediador tiene la intención de lograr que el alumno aprenda, a que el aprendizaje le sirva no solo para el caso específico sino también para otras situaciones similares; se asegura de que profesor y estudiante tengan un propósito común y que entiendan las cosas de la misma manera, que recalque el avance del aprendiz hacia la meta, que ayude a que no se desvíe del propósito y que la actividad de aprendizaje la realicen entre ambos.

Así, el desarrollo cognoscitivo de los alumnos ocurre a través de una serie de transformaciones cualitativas, asociadas con cambios en el uso de los instrumentos físicos y psicológicos propios de la cultura a la que pertenece el aprendiz. Las funciones psicológicas superiores aparecen en dos dimensiones distintas: en un primer plano social o interpsicológico y, posteriormente, en el plano individual o intrapsicológico, pero no como producto de un proceso de imitación o copia, sino de uno de internalización progresiva, de un proceso reconstructivo (Vigotsky, 1979). Es decir, las funciones pasan de llevarse a cabo entre quienes ya lo conocen, en este caso, la profesora y un aprendiz quien inicialmente las ejecuta junto con la mediadora, hasta que este pueda llevarlas a cabo internamente y las pueda ejecutar sin ayuda. 
A partir de lo anterior, se puede comprender que el aprendizaje solo ocurre en la zona del desarrollo próximo y que es justo ahí donde el alumno requiere del apoyo del profesor. Una vez que el niño ha comprendido un tema o internalizado un procedimiento, puede trabajar de manera independiente para dominarlo. Ya no requiere la ayuda de otro; simplemente, practicar o procurar relacionarlo con otros conocimientos.

Lo central en este enfoque constructivista consiste en la organización del trabajo de los aprendices en torno a actividades significativas en las que se involucren concienzudamente. Es decir, que los alumnos revisen su propia experiencia de aprendizaje, que consideren múltiples maneras de visualizar un asunto, que ayuden a planear las actividades de clase y diseñen o resuelvan problemas en donde no hay respuestas correctas de antemano.

De acuerdo con Lerner (citado por Hernández, 2000), desde el enfoque constructivista, un maestro plantearía situaciones problemáticas que demanden y favorezcan en los alumnos un trabajo reconstructivo de los contenidos, lo cual debe suponer una distancia cognoscitiva entre el problema y su capacidad, de manera que provoque leves desajustes y los anime a elaborar nuevas y más ricas interpretaciones. Además, promovería situaciones de diálogo en donde se intercambien puntos de vista acerca de problemas académicos o morales, aportaría la información que requirieran los estudiantes para la reconstrucción de los contenidos escolares, y examinaría y cuestionaría las opiniones de los alumnos a lo largo del proceso educativo.

Si se desea favorecer el aprendizaje, una idea central sería la de construir escenarios de actividad que lo posibilite, los cuales no están constituidos por el espacio físico y el mobiliario, sino principalmente por el profesor y los alumnos, con toda la riqueza de interacciones que ocurren entre ellos, así como por la meta de esas actividades conjuntas. Estos escenarios diversificados tienen un impacto en la calidad de la relación entre profesor y alumnos, y entre estos, y que repercuten en el aprendizaje o en la falta de este.

Regresando al problema de la falta de desarrollo de la literacidad, ya que este inicia en los grados escolares tempranos, se decidió hacer este estudio con niños pequeños que presentan dificultades con la lectoescritura inicial. Uno de los principales predictores del rendimiento en lectura es el nivel socioeconómico (OCDE, 2019), por lo que se realizó el estudio en un grupo que perteneciera a un estrato bajo y que, además, presentara dificultades para desarrollar habilidades para leer y escribir. Los niños del grupo elegido son referidos por las escuelas del sur de la Zona Metropolitana de Guadalajara a un centro de atención educativa, debido a que presentan fallas en la adquisición de la lectoescritura inicial. Es decir, se conjuntan dos situaciones de vulnerabilidad: el bajo nivel socioeconómico y dificultades personales para el aprendizaje de esta competencia.

La investigación se realizó en el Centro Polanco, una institución que atiende a niños de entre 6 y 12 años que presentan dificultades en la apropiación de la lectura y la escritura, entre otras. Uno de los requisitos del centro es que estén inscritos en la primaria, ya que el apoyo que 
brinda es extraescolar y pretende fortalecer los aprendizajes que adquieren en la escuela, pero no la sustituye. Los profesores de las escuelas de la zona y los padres de familia refieren avances importantes en el aprendizaje de los niños cuando asisten a este centro.

El objetivo de la investigación fue caracterizar y describir aquellas acciones mediacionales que ayudan a los niños en el desarrollo de la competencia de literacidad, pues al identificarlas y describirlas, otras personas del mismo centro o de otros, podrán beneficiarse al hacer uso de aquellas que fueron más eficaces.

\section{Método}

De acuerdo con el interés de la investigación se eligió como método el estudio de casos, debido a que permite analizar con profundidad un fenómeno delimitado, dar cuenta de las acciones e interacciones en relación con los contextos (Stake, 1999) y comprender con ello las acciones mediacionales que utilizan las profesoras involucradas en el estudio para favorecer el aprendizaje en circunstancias concretas y particulares. Este método posibilita la inclusión tanto de los aspectos objetivos como subjetivos de las prácticas que se estudian (Neiman, Quaranta, 2012), en este caso las de literacidad. Consideramos el caso como la entidad completa y diferenciada constituida por los participantes, el entorno, las herramientas que se utilizan, las interacciones y los objetivos que persiguen, pero este artículo se focaliza en la mediación.

El lugar en que se llevó a cabo la investigación es el Centro Polanco, un escenario de acción social comunitaria que brinda servicios psicoeducativos a los habitantes de colonias populares del sur de la Zona Metropolitana Guadalajara, en donde estudiantes de distintas licenciaturas del Instituto Tecnológico y de Estudios Superiores de Occidente (ITESO) realizan prácticas profesionales en las que ejercitan lo aprendido durante su formación inicial y, al mismo tiempo, dan un servicio que responda a las necesidades educativas que presenta una población específica. En las prácticas de literacidad participaron dos alumnas de los últimos semestres de las carreras de Psicología y Educación, quienes fungieron como facilitadoras de los procesos de aprendizaje. Ambas recibieron entrenamiento previo, por parte de la coordinadora del centro, acerca del enfoque constructivista sociocultural, habilidades fonológicas, principio alfabético y la teoría que sustenta la enseñanza de la lectoescritura inicial.

En este proyecto participaron ocho niños, además de las facilitadoras; tres fueron remitidos al centro por profesoras o la directora, los cinco restantes acudieron por recomendaciones que vecinas y familiares hicieron a las madres de familia. El motivo de solicitud de atención fue que los niños no habían aprendido a leer y a escribir. De los ocho niños, tres cursaban el primer año de primaria, dos el segundo y tres el tercero. Uno de los niños está diagnosticado con Asperger. La edad promedio del grupo fue de 7 años. El promedio de asistencia fue de 6.6 estudiantes por sesión. Los niños provienen de familias en donde los padres tienen escolaridad baja, que va desde no escolaridad hasta preparatoria como máximo. 
Los niños fueron evaluados antes y después de la intervención con dos pruebas: 1) Indicadores Dinámicos del Éxito en la Lectura (IDEL) (Baker, Good, Knutson, Watson, 2006), una prueba individual estandarizada que identifica el nivel de riesgo que un alumno tiene para desarrollar las destrezas lectoras evaluando cinco habilidades fundamentales para la lectoescritura, y 2) La prueba de lectura y escritura elaborada por el programa del centro, basada en la propuesta pionera de Ferreiro y Gómez Palacio que, desde una mirada psicogenética, explica cómo los niños van apropiándose de este sistema de representación. Desde esta perspectiva, el aprendizaje de la lectura y la escritura es visto como un proceso evolutivo que implica el tránsito del aprendiz por una serie de estadios cualitativamente diferenciados (Gómez-Palacio, Cárdenas, Guajardo, Kaufman, Maldonado, Richero, Velázquez, 1984), que inicia con el conocimiento fonológico y sintáctico, y finaliza con el conocimiento semántico y pragmático.

Aunque la finalidad de este estudio no fue evaluar los resultados sino describir el proceso de mediación utilizado por las profesoras, se muestran algunos datos para dar idea de cómo llegaron los niños y cómo estuvieron al final. Por ejemplo, en uno de los indicadores de la prueba IDEL, Fluidez en la Lectura de Palabras, al inicio, leían un promedio de cinco por minuto y al terminar el periodo ya leían 16 .

En la prueba de lectura y escritura, al inicio del curso, la escritura predominante del grupo era la presilábica. Los niños escribieron un total de 42 palabras clasificadas bajo esta categoría, seguida por la escritura de 20 palabras silábicas, 10 palabras silábicas alfabéticas, 2 palabras alfabéticas cortadas y únicamente 3 palabras escritas alfabéticamente. Los resultados de la segunda evaluación muestran un incremento en la escritura de palabras alfabéticas: de 3 aumentaron a 14 y hubo una disminución en el número de palabras escritas bajo la hipótesis presilábica; de 42 disminuyeron a 14, siendo la escritura silábica alfabética la predominante en el grupo, con un total de 23 palabras escritas bajo esta categoría. Puede notarse que hubo avances en ambas mediciones. El tiempo de intervención fue corto, pero los niños continuaron siendo atendidos después de estas evaluaciones.

El aprendizaje de la lectoescritura se llevó a cabo en 16 sesiones de 80 minutos durante el primer semestre de 2019, dos veces a la semana, conducidas por las dos facilitadoras. Todas las sesiones fueron videograbadas y transcritas. El tamaño del grupo variaba entre cinco y ocho niños debido a que no siempre asistían todos. Las prácticas fueron analizadas con un doble propósito: identificar las que producen cambios microgenéticos (que ocurren en un lapso de tiempo muy corto) en la competencia lectora, y contar con elementos que permitan ajustar el tipo de ayuda que se está proporcionando a los niños para que continúen aprendiendo. Con el fin de cuidar la confidencialidad, en el análisis se omiten los nombres de las facilitadoras y los de los niños fueron cambiados.

Las viñetas que se incluyen para ilustrar el análisis fueron tomadas textualmente de las transcripciones; cuando son cortas se ponen entre comillas, y cuando son largas se incluyen como párrafos adicionales con sangría. 
Los datos cualitativos se fueron analizando a la par de la intervención. Para el análisis de los datos provenientes de las observaciones transcritas se siguió la propuesta de Miles y Huberman (1994): reducción de datos, despliegue e interpretación. Para la reducción se codificaron en el programa Atlas $\mathrm{Ti}$, luego se desplegaron en matrices $\mathrm{y}$, a partir de ellas, se identificaron y describieron las distintas acciones mediacionales. El proceso analítico se realizó de manera inductiva y los datos se interpretaron desde el marco de la teoría sociocultural.

\section{Niños, letras y mediaciones}

Durante las sesiones se trabajó principalmente en que los niños reconocieran grafías, identificaran fonemas, realizaran operaciones de análisis y síntesis fonológico, así como en el desarrollo del lenguaje. Por lo general se trabajaba con el grupo completo, pero dando atención a las necesidades particulares que presentaba cada niño. En ocasiones los niños trabajaban en parejas o triadas. Una característica notoria fue que el trabajo se llevó a cabo en un ambiente cordial en el que no se observó que se reprendiera a los niños, más bien se les invitaba a trabajar, se animaba la participación y se reconocían sus aportaciones.

Se aclara de inicio que al terminar el periodo de intervención no se observaron cambios espectaculares en el dominio del lenguaje escrito por parte de los niños. Los cambios fueron modestos y diferentes en cada uno de ellos, lo cual llama la atención si se toma en cuenta el trabajo cuidadoso y metódico que hicieron las facilitadoras. A pesar de que no se haya logrado un avance mayor en el dominio de la lengua escrita por parte de los aprendices, es importante caracterizar las acciones mediacionales utilizadas por las enseñantes debido a que tienen el potencial de generar aprendizajes significativos si se hacen las modificaciones que se proponen a partir del análisis realizado.

Para favorecer las habilidades lectoras iniciales, las facilitadoras despliegan una amplia gama de acciones mediacionales que fueron utilizadas con distinta frecuencia, como puede apreciarse en el gráfico 1. 


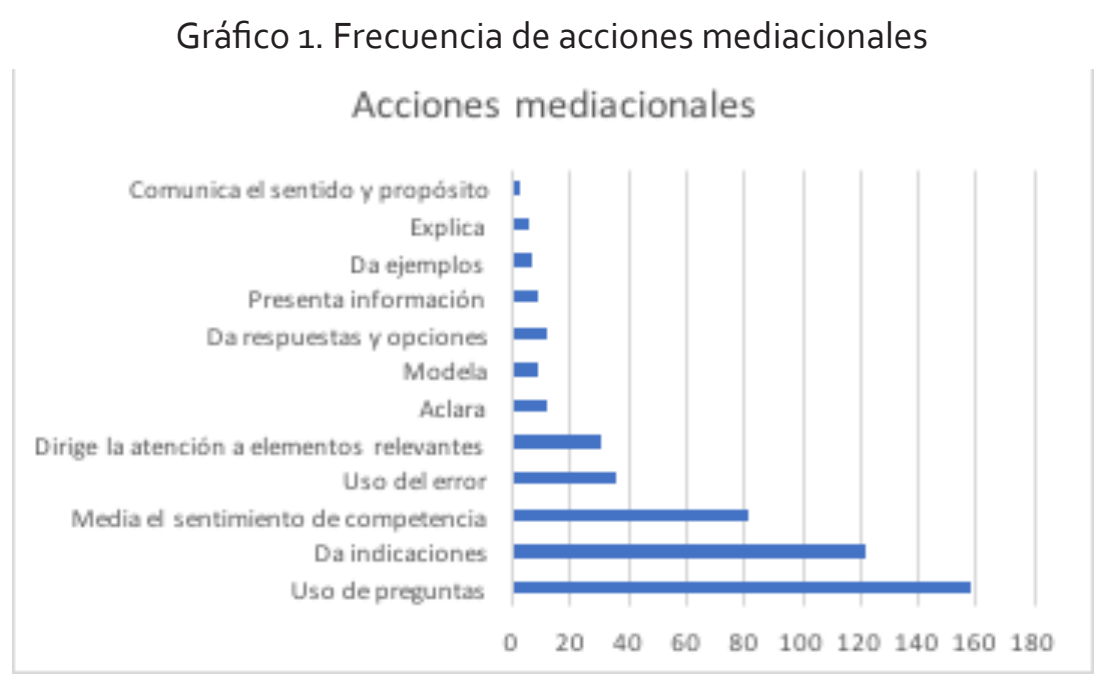

Se identificaron tres grandes categorías. La primera relacionada con I. dar sentido a la actividad y fortalecer el sentimiento de ser competente para realizar la tarea. Otro conjunto de acciones tiene que ver con II. la enseñanza directa. La tercera refiere a la intención de III. favorecer los propios procesos reflexivos de los niños que les permitan aprender directamente a partir de sus acciones. Buena parte de estas acciones mediacionales ocurrieron en la zona del desarrollo próximo de los aprendices, es decir, sirvieron para que los niños tuvieran un desempeño que no podrían haber logrado solos, pero sí con la ayuda de las facilitadoras. Cada una de las categorías agrupa diversas acciones mediacionales, las cuales se caracterizan en las secciones siguientes.

\section{El sentido de la actividad y la retroalimentación del desempeño}

De acuerdo con Haywood, Brooks y Burns (1991), dos de las características de la mediación eficaz son: 1) comunicación del sentido y propósito, y 2) la mediación del sentimiento de ser competente. La primera sirve para que el niño sepa el qué y el para qué de lo que va a hacer, que en este trabajo se define de la siguiente manera: la mediadora comparte con el niño el para qué de una actividad y señala su relevancia. Este elemento es el gran ausente en el trabajo que hacen las facilitadoras, pues aparece en pocas ocasiones. Aunque ellas tengan preparada la clase, dispuestos los materiales y sepan lo que van a hacer, con frecuencia el propósito no es comunicado a los niños.

En el transcurso del semestre hubo tres ocasiones en que se comunicó el sentido de la actividad. La primera fue cuando se trabajó con los niños en la elaboración de un pastel siguiendo una receta. A partir de la actividad se dialogó sobre la importancia de la lectura para una actividad cultural importante: la preparación de alimentos. En otra, mientras los participantes están trabajando en grupos de tres para formar palabras, la facilitadora señala el propósito de la actividad cuando ve que un niño quiere competir para ganar: 
La facilitadora dice a Pepe que ayude a una pareja de niños para que sean tres. Él rápidamente se para de su lugar y se va a un lado de ellos. Alex menciona que hay que ganar. La maestra le dice que no es de ganar, que el chiste es que aprendan cómo se escribe.

Y en la tercera ocasión, la facilitadora informa a los niños que van a pintar sus manos en un papel, aunque aquí no les dice el propósito o el sentido:

Facilitadora: "pues lo que vamos a hacer ahorita que salgamos, vamos a salir en orden, vamos a pintarnos nuestras manos con pintura con mucho cuidado de no mancharnos, así, con los dedos separados los vamos a poner en un papel" (Mientras pone las manos sobre la mesa).

Otra mediación importante para el aprendizaje de los niños es la que fomenta en los niños el sentimiento de que son competentes para realizar la actividad (Haywood, Brooks, Burns, 1991), lo que en este caso ocurre con la retroalimentación positiva que dan las facilitadoras acerca de su desempeño. Esta fue el tercer tipo de intervención más utilizada, con 81 incidencias, solo después de dar indicaciones, que tuvo 122, y uso de preguntas con 158. La importancia de retroalimentar positivamente el desempeño de los niños reside en que esta acción constituye una manera de informar que su intervención es correcta o valiosa, lo cual puede ayudar a promover la participación y a mejorar su desempeño.

A pesar de la incidencia alta de este tipo de intervención, el análisis muestra pocas variaciones en su uso y en su forma. Por lo general se retroalimenta a un niño; "ajá, muy bien Toño", pero ocasionalmente también se retroalimenta al grupo: "Muy bien, Simón dice que todos nos demos un aplauso". Se tiende a retroalimentar las respuestas correctas; "iMuy bien! Entonces Pelota y Perro sí comienzan igual"; a los productos: Diana muestra su hoja con la actividad y la maestra le dice "Sí, muy bonito, qué hermoso"; a las actividades terminadas:"ya acabaste todo el cuento, a ver, vamos a ver. Uy muy bien"; velocidad de trabajo: "Ándale muy bien. ¡Qué rápido!"; y un proceso parcial en que el niño va bien: "vas muy bien, solo que esa parte era para doblar".

La retroalimentación positiva puede ser tácita: "Oye Darío, sí es cierto, la letra número 4 es la A" o puede ser explícita: "muy bien Paty, muy bien". Se retroalimenta con elogios, aplausos y en ocasiones se incluye algún premio como un chocolate, una pegatina o la realización de una actividad, como que la niña tenga el privilegio de escribir una palabra: "Diana va a ayudarme a escribir porque está poniendo mucha atención. Diana, escribe 'esa' en el pizarrón".

Estas dos acciones mediacionales, comunicar el sentido de la actividad y retroalimentar a los niños acerca de que son competentes para realizarla, son importantes para lograr que estos se involucren y perciban un ambiente apropiado para el aprendizaje, lo cual es necesario para que, a partir de eso, vayan desarrollando el dominio del código escrito para la comunicación y la participación en las actividades socioculturales que lo requieren. 


\section{La enseñanza directa}

En este trabajo, la enseñanza directa se define como las acciones mediante las cuales la profesora presenta a los niños aquella información que, a su juicio, deberán aprender. En esta categoría se incluyen las acciones mediacionales en que la profesora se interpone entre el aprendiz y el objeto de conocimiento, funcionando en parte como base de datos y en parte haciendo la información accesible al niño. Estas incluyen 3) presentar información, 4) dar indicaciones, 5) explicaciones, 6) modelamiento, 7) aclaraciones, 8) ejemplos y 9) respuestas y opciones de respuesta.

Presentar información, como estrategia mediacional, se utilizó poco durante las sesiones de desarrollo de la literacidad en los niños, pues apareció únicamente en nueve ocasiones. Este tipo de intervención se utiliza para ayudar a los alumnos a que adquieran conocimiento que deberán tener en mente o utilizar en la actividad inmediata. Tal contenido no requiere gran comprensión ni profundización.

En ocasiones, se presenta información para enseñar a los niños a realizar una actividad simple, por ejemplo, la manera de articular un sonido, como en la siguiente viñeta: "Esta lámina es sobre la letra $A$, entonces aquí nos explica cómo se hace la boca con la $A$ ise acuerdan que la vieron en el espejo?". También se utiliza para que los alumnos conozcan alguna regla, como en el caso que se presenta a continuación, en el que para jugar memorama se les indica de qué se trata: "Ah, pues les vamos a explicar. La memoria o memorama trata de que por turno tienen que voltear dos fichas"; así como para dar un dato específico, como la localización o alguna característica de un país: "este libro lo escribieron en 2013, en ese año lo escribieron y lo imprimieron en España [...] España es un país como México, que está en Europa y en donde hablan español también".

Presentar información, además se utiliza cuando los niños pronuncian de manera incorrecta una palabra, por ejemplo, cuando un niño muestra a otro cómo hacer un sonido y menciona: "ira, asi", la profesora lo corrige diciendo "mira"; o cuando se equivocan en nombrar algo. Un niño dice que van a poner frijol, cuando que el material que están utilizando es pasta, la profesora pregunta: ¿A echarle frijol?" Se ríe y dice: "esto no es frijol, es pasta". En estos casos la profesora proporciona información precisa.

Otra de las formas de mediación que utilizaban frecuentemente las facilitadoras fue dar indicaciones, lo cual refiere a decir a los niños de manera precisa qué y cómo hacer para realizar una actividad. Esta es una de las mediaciones más utilizadas pues aparece en 122 ocasiones. La mayor parte del tiempo se utiliza para describir las tareas que se solicitan a los miembros del grupo, por ejemplo: "ahorita lo que vamos a hacer, es que les voy a dictar unas palabras y ustedes las escriben como puedan, ¿sale? ¿listos? las van a escribir así en hilerita". Un ejemplo más es el siguiente: "Ahora vamos a dibujar en la silueta las cosas que nos gustan de nosotros".

Aunque el mayor uso que tiene el dar indicaciones es para ayudar a que realicen las actividades que se llevarán a cabo dentro del salón de clase -se registró un total de 78 indicaciones 
de este tipo-, este medio se utiliza también para ayudar a los niños en su desempeño, cuidar la organización y clima de grupo, así como la administración del salón, como se verá a continuación.

Para apoyar el desempeño de los niños, la instructora da indicaciones precisas a un niño en particular. Se acerca a este y con un lápiz le señala mientras dice:"a ver, te voy a poner líneas, ¿sale?"; los anima a participar: "Si quieres hazte más para acá para que nos ayudes a ponerlas" y marca el ritmo de trabajo del grupo: "rápido, Elena, que vamos a pasar a la siguiente" o "¿listo Fausto? rápido".

Para la organización y el manejo del clima de grupo la profesora indica a los niños la manera en que tendrán que agruparse para trabajar: "vamos a hacer dos equipitos. Carlos, ponte en esa silla de allá" o"van a ser un equipo Paulina y Darío"; cuida el clima afectivo, por ejemplo, presentando a un niño recién llegado: "van a decir sus nombres para Fausto" y regula la conducta de los participantes señalando los acuerdos de convivencia establecidos o dando indicaciones sobre conductas específicas: "siéntense en sus sillitas para hacer el cierre".

Las facilitadoras también utilizan las indicaciones para cuidar aspectos de manejo del salón, entre ellos, para que ordenen y guarden los materiales y para que registren su asistencia. Esto ocurre en todas las sesiones, lo que ayuda a que el salón permanezca ordenado y a que quede constancia de la asistencia de cada niño, sin que ellas tengan que encargarse de eso, pues los niños registran directamente su asistencia en una cartulina en donde está la lista de todos los participantes, lo que a la vez les permite identificar su nombre una y otra vez.

Una de las acciones de mediación que utiliza la instructora es la de explicar. Esta acción fue definida como presentar de manera clara una idea o elaborar sobre esta, con la finalidad de que el niño comprenda. Ocurrió únicamente en seis ocasiones, por tanto, es una de las que tienen menor frecuencia, solo hay una por debajo de ella con menor número de apariciones, como se mostró en la gráfica 1.

La profesora utiliza la explicación para que un niño comprenda el funcionamiento de un objeto que aparece en uno de los libros que se utilizan durante la enseñanza, un embudo: "Se usa cuando tienes mucho de algo, por ejemplo, gasolina o azúcar, o agua, y quieres ponerlo en un lugar más chiquito, entonces pasa por aquí", mientras señala el cuello del embudo en la ilustración de la tarjeta. También la usa cuando en una de las narraciones se presenta una relación complicada: "la madre de Karla se ha casado por tercera vez, o sea, se casó una vez, luego se casó otra vez, luego se casó una tercera vez, pero ella nació del segundo matrimonio de su madre".

La explicación también sirve para ayudar a que los niños comprendan la diferencia entre dos conceptos que tienen similitudes, tanto en su uso como en su nombre. En este caso se explica el uso o función del telescopio, debido a que un niño lo confundía con el microscopio. Este último, como concepto, apareció durante la enseñanza. Así lo dice la instructora: 
con el que vemos la luna es un telescopio, el telescopio es uno así grandote, largo, así, aquí está la personita y el telescopio es como algo así, grandote para ver por ejemplo la luna (la facilitadora se levanta de la silla, se dirige al pizarrón, toma un plumón, se pone en cuclillas y en el pizarrón dibuja una persona con un telescopio).

Finalmente, la explicación se usa para definir conceptos abstractos cuyo significado no es conocido por los niños, como en el siguiente caso: "La palabra perceptible significa que es tan pequeñito que apenas lo podemos ver. Como con una lupa, como está ahí (señalando la ilustración del cuento)".

Una acción mediacional más es la aclaración, que la maestra utiliza cuando un alumno ejecuta una acción de manera equivocada por no haber comprendido una instrucción. En este caso da otra instrucción con más detalle y precisión. Al ver que el niño pega una letra en el lugar que no corresponde, le da una indicación más precisa: "esta la vamos a despegar de acá y la vamos a pegar acá abajo. Ahí va, es el primer sonido de pato ¿sale?".

Cuando los niños confunden el significado de una palabra con otra, microscopio con telescopio, aclara proporcionando mayor información: "aquí digamos esta personita sentada y esta es su mesa y el microscopio puede estar chiquito y es para ver cosas chiquitas ¿sí? Y ahí se asoma la persona y las cosas que son las hace más grandes". Ante la aclaración, una niña muestra su comprensión comparando el microscopio con algo que ella ya conoce: "como la lupa".

La aclaración como acción mediacional fue importante porque ayudó a los niños a corregir sus errores para tener un conocimiento más preciso. Valga señalar que todas las aclaraciones fueron hechas con una actitud de respeto, ante lo que los niños no parecían sentirse incómodos ni se inhibía su participación.

Un recurso mediacional más, del cual echan mano las profesoras es el modelamiento, es decir, ellas realizan la actividad para que los niños observen y a su vez puedan repetirla. Se utilizó en nueve ocasiones, la mayor parte del tiempo es para que los niños sepan identificar corporalmente cómo articulan los fonemas. Muestra cómo pone la boca para producir un sonido en particular y lo alarga; luego pide que los niños lo repitan, enseguida les pide que observen en el espejo cómo ponen los labios y la lengua para producir los sonidos.

El modelamiento también lo utiliza para que aprendan más rápido a utilizar los juegos didácticos como el memorama de palabras que se ve en el siguiente ejemplo: "A ver, lo volteo y veo la A y la L, ¿son iguales?" Niños: "No". Va el siguiente:"Imagínense que el siguiente voltea A y luego A y dice AA (hace el sonido) árbol y abeja. Entonces se queda con ese par ¿de acuerdo?".

Dentro de esta categoría, la última acción mediacional es dar respuestas y ofrecer opciones cuando los niños no saben responder. En pocas ocasiones los alumnos formulan preguntas y las facilitadoras contestan de manera directa. Por ejemplo, ella dice la palabra "unión" y los niños preguntan qué es eso, a lo que contesta: "Unión es porque están juntas, están pegadas" o"¿Qué es ubre? Donde sacamos la leche de la vaca". 
Fue más frecuente que la profesora ofreciera opciones de respuesta, ya sea a nivel grupal o individual, con las finalidades de pedir precisión en sus respuestas, Ilamar la atención hacia un elemento relevante o apoyar en la realización de una actividad. Fueron 13 ocasiones en que se utilizó esta acción mediacional. En el siguiente ejemplo se pueden ver las opciones de respuesta cuando les pregunta cómo articular el sonido de la letra P:

Facilitadora 1: ¿entonces cómo están los labios?

Elena: bien.

Facilitadora 2: ¿cómo los tenemos que hacer para decir P?

Elena: así, mientras cierra sus labios.

Facilitadora 1: ¿separados o juntos?

Alumnos: juntos.

Facilitadora 2: ¿abiertos o cerrados?

Paty: cerrados.

Facilitadora 2: cerrados ¿verdad? Sí, muy bien.

Se observó que las acciones mediacionales de presentar información, dar indicaciones, explicar, modelar, aclarar, dar ejemplos, respuestas y opciones de respuesta, a pesar de estar centradas en las profesoras, ayudan a los alumnos a desarrollar algunas de las habilidades básicas como identificación de grafías, reconocimiento fonémico, análisis y síntesis de palabras que les permitirán construir la competencia de literacidad y se complementan con otras que están más centradas en que los niños aprendan a partir de sus acciones y experiencias.

\section{Fomento de los procesos reflexivos}

En la categoría de fomento de los procesos reflexivos se agrupan las acciones mediacionales que tienen la intención de favorecer que los niños construyan aprendizajes a partir del descubrimiento de patrones, de notar inconsistencias o de hacer inferencias inductivas. Esto incluye: 10) llamar la atención de los niños a los aspectos relevantes de lo que deben de aprender, 11) las preguntas que tienen el propósito de ayudar a los alumnos a aprender directamente a partir de sus acciones, y 12) el uso del error como elemento para el aprendizaje.

Aunque las mediaciones incluidas en la categoría de enseñanza directa son muy relevantes para que los niños aprendan, estas que fomentan los procesos reflexivos son de importancia capital debido a que los estudiantes aprenden por sí mismos, a partir de su propia experiencia con la ayuda de las profesoras.

Cuando las personas aprenden un proceso o contenido nuevo, se enfrentan a un cúmulo de información que es percibida por ellos como amorfa y no saben a qué prestar atención. En este punto interviene el mediador a través de la acción de dirigir la atención de los aprendices hacia aquellos estímulos de la información que son relevantes para el aprendizaje; sin esta focalización sería muy difícil o imposible tener aprendizajes específicos. 
Como parte del análisis fonológico, el aprendiz necesita identificar los sonidos que conforman una palabra, por lo que la profesora dirige la atención primeramente a la presencia o ausencia de un fonema: "elefante ¿tiene F?". El niño responde que sí, enseguida le pregunta:" ¿al principio, en medio o al final?" El niño responde que "en medio", la facilitadora valida la información y reconoce el logro: "en medio, muy bien". En esta secuencia puede observarse cómo dirigir la atención del niño a los aspectos relevantes, le va permitiendo desarrollar sus habilidades fonológicas, indispensables para el desarrollo de la competencia lectora (Shaywitz, 2003). No es una información que la profesora pueda proporcionar, sino un conocimiento que el niño debe generar a partir de la identificación de los fonemas que componen la palabra. No lo puede hacer solo sino con la ayuda de la profesora, por lo que es un caso muy claro de mediación en términos vigotskianos y de ayuda en la zona del desarrollo próximo.

La acción mediacional de dirigir la atención hacia aspectos relevantes tiene distintos usos, como puede verse en la tabla 1. En esta se muestran los nueve usos más frecuentes y se ilustran con ejemplos concretos.

Tabla 1. Mediación: dirigir la atención hacia aspectos relevantes

\begin{tabular}{|l|l|}
\hline \multicolumn{2}{|c|}{ Las facilitadoras dirigen la atención de los niños hacia aspectos relevantes } \\
\hline Uso & Ejemplo \\
\hline $\begin{array}{l}\text { Identificar la presencia de } \\
\text { un fonema }\end{array}$ & $\begin{array}{l}\text { Facilitadora: ¿y la palabra fuente lleva el sonido de la F (haciendo } \\
\text { el sonido)? ¿Sí o no? } \\
\text { Niños: sí }\end{array}$ \\
\hline $\begin{array}{l}\text { Establecer semejanzas en- } \\
\text { tre sonidos }\end{array}$ & $\begin{array}{l}\text { Facilitadora: ¿Qué tienen en común estas tres palabras? } \\
\text { Paty: A } \\
\text { Facilitadora: A, ándale, vale, que todas tienen la A. }\end{array}$ \\
\hline $\begin{array}{l}\text { Ubicar el lugar que ocupa } \\
\text { una letra dentro de una } \\
\text { palabra }\end{array}$ & $\begin{array}{l}\text { Facilitadora: ¿Dónde tiene la P mariposa? ¿al principio, en medio } \\
\text { o al final? } \\
\text { Niños: En medio. }\end{array}$ \\
\hline $\begin{array}{l}\text { Descubrir la manera de arti- } \\
\text { cular un sonido }\end{array}$ & $\begin{array}{l}\text { Mara: A-ra-ña. } \\
\text { Facilitadora: ¿Viste cómo tu boca hizo tres veces así? (mientras } \\
\text { abre la boca). }\end{array}$ \\
\hline $\begin{array}{l}\text { Identificar objetos en imá- } \\
\text { genes }\end{array}$ & $\begin{array}{l}\text { Facilitadora: ¿qué más hay aquí? (señala la hoja con el dedo } \\
\text { índice). } \\
\text { María: Pelota } \\
\text { Facilitadora: ¿Y qué más hay aquí? } \\
\text { Darío: Una cama } \\
\text { Facilitadora: ¿Qué es esto, Darío? } \\
\text { Darío: Una pizza. }\end{array}$ \\
\hline Descubrir el error & $\begin{array}{l}\text { Facilitadora: ¿Cuál es el primer sonido de gato? } \\
\text { Toño: Ga (lo repite varias veces) } \\
\text { Facilitadora: (pronuncia ggg) y replica que ga son dos sonidos g- } \\
\text { a y ahorita queremos solo uno, el primero. }\end{array}$ \\
\hline
\end{tabular}




\begin{tabular}{|l|l|}
\hline $\begin{array}{l}\text { Identificar fonemas faltan- } \\
\text { tes }\end{array}$ & $\begin{array}{l}\text { Facilitadora:Y después ¿qué sigue? Junto con la facilitadora repi- } \\
\text { ten r-a-t-o } \\
\text { Facilitadora: ¿Ya acabamos de escribir ratón o falta algo? } \\
\text { María: (grita) la ene } \\
\text { Facilitadora: La nnn. }\end{array}$ \\
\hline $\begin{array}{l}\text { Distinguir entre grafías si- } \\
\text { milares }\end{array}$ & $\begin{array}{l}\text { Paulina coloca fichas para completar la palabra embudo, pero la } \\
\text { que forma es embubo. } \\
\text { Facilitadora: (se acerca y señala la b) y dice ¿esta es la d? } \\
\text { María: Quita la ficha de la b y la coloca en la caja, toma una y la } \\
\text { pone en su lugar. } \\
\text { ¿Esta y este son iguales? (subraya la letra d y b en el pizarrón) } \\
\text { Niños: No } \\
\text { Facilitadora: No, ¿verdad? ¿Para dónde tiene la pancita la d? ¿Para } \\
\text { la derecha o la izquierda? } \\
\text { María: izquierda } \\
\text { Facilitadora: Izquierda, ¿ya vieron? }\end{array}$ \\
\hline Distinguir minúsculas y ma- \\
yúsculas & $\begin{array}{l}\text { Facilitadora: Niño. Nnn ¿ya vieron? Así fueran mayúsculas sería } \\
\text { NIÑO y con la misma N. (escribe Niño en el pizarrón) } \\
\text { Mara: ¿cuál es la N mayúscula? } \\
\\
\text { María: Ésta (señala con el dedo N en pizarrón). }\end{array}$ \\
\hline
\end{tabular}

Llamar la atención hacia los aspectos relevantes de los objetos de aprendizaje es primordial debido a que ante una enorme gama de estímulos que perciben los niños no sabrían a cuáles de ellos poner atención, a menos de que un adulto que sabe cuáles son aquellos a los que hay que atender para aprender a leer y escribir, se los señale.

Uno de los recursos mediacionales más utilizados por la profesora fue el de la pregunta para recuperar información, conocimientos y experiencias, apareció en 158 ocasiones. Estas preguntas guía llevan a los niños a que utilicen su conocimiento previo para potenciar su aprendizaje. El análisis revela que fueron seis los principales usos: a) identificar y reconocer un elemento, suceso u objeto; b) ayudar a que los niños se apropien del objeto de conocimiento; c) aplicar o relacionar lo aprendido; d) distinguir las partes y relacionarlas con la estructura global; $e$ ) evaluar; $y$ f) favorecer la colaboración.

Para recuperar información la facilitadora pregunta: ¿Qué día es hoy? Hoy es el día del...." Un niño contesta: "Día del amor".

En otra ocasión la facilitadora ayuda mediante preguntas a que el niño recuerde elementos de la historia leída:

Facilitadora: ¿Qué está haciendo el niño?

Niño: Buscando.

Facilitadora: ¿Por dónde lo está buscando?

Niño: Por todas partes. 
Facilitadora: "y ¿dónde está Fabio?

Niño: En un gorro mágico.

Durante el trabajo se puede observar una gran cantidad de ocasiones en que los niños no tienen en mente alguna información y la facilitadora les ayuda a recuperarla mediante preguntas. Las preguntas pueden servir para identificar algún elemento, suceso u objeto, como cuando la instructora cuestiona: “y qué más ven ahí en la foto?". Los niños responden que es una pala, ella inquiere: “dónde están los niños?", señalan con la mano el lugar, enseguida, ella los interroga sobre la utilidad de las palas y los niños responden que son para cavar. Aquí los estudiantes identifican un elemento y lo relacionan con su utilidad. La maestra también usa la pregunta para evocar experiencias de los alumnos: ¿Qué hicieron el fin de semana?", “¿A dónde fueron?".

La pregunta también sirve para recuperar información que está en la mente de los niños, pero que no han expresado completamente. Con frecuencia, estos dan información parcial y asumen que sus interlocutores conocen el resto. Ante este tipo de situaciones, la facilitadora formula preguntas como las siguientes: “YY qué más pasa en tu historia? ¿En qué termina tu cuento?, platícanos". Un uso más de esta acción mediacional es que el aprendiz aplique el conocimiento que ya tiene, por ejemplo, para que identifique el título de un libro: “¡Y cuál será el título del libro?"; que lean una palabra escrita: “Qué dice ahí, Carlos?"; para hacer el análisis fonémico de una palabra: “Cuántos sonido nos faltan?". En todos estos ejemplos los niños tienen que poner en juego el conocimiento que ya poseen para dar respuesta a la interrogante planteada por la profesora.

Las preguntas son utilizadas para que los alumnos identifiquen partes y las relacionen con el todo, por ejemplo, una vez que el niño dice que la palabra amistad tiene el fonema "a", la maestra pregunta en dónde lleva otra "a" esa palabra. Para practicar el uso de ese mismo fonema los interroga sobre cuántas "a" tiene la palabra mamá. Al estar formando una palabra les cuestiona: “Qué otra letra necesitamos?" O “cuál es el primer sonido de la palabra pato?”. En todos estos ejemplos se trata de que los niños identifiquen las partes (fonemas) y la relacionen con el todo (palabras).

Las preguntas pueden evocar comentarios evaluativos como en los siguientes casos: ¿Qué piensan de los acuerdos del día de hoy? ¿Los respetamos?”, ¿Fuimos amables? ¿Cómo sabemos que fuimos amables?". Finalmente, se encontraron algunas preguntas que van encaminadas a favorecer la colaboración entre aprendices. En una ocasión la instructora pregunta, ¿Quién le puede decir a Carlos qué sonido estamos viendo?", ¿Quién dice a Carlos cómo tenemos que hacer nuestra boca para hacer el sonido?". En estos casos la intención de la facilitadora es que los aprendices colaboren en el aprendizaje de sus compañeros en lugar de hacerlo ella misma.

El uso del error es un medio de ayuda que, de manera sistemática y constructiva, utilizan las facilitadoras a lo largo de todas las sesiones de trabajo. En el transcurso de una actividad, un 
error presenta la oportunidad de provocar procesos reflexivos tanto a nivel grupal como individual, que permiten al colectivo o al sujeto en particular encontrar la respuesta correcta al darse cuenta de la equivocación cometida, recapacitar sobre ella y corregirla. En este trabajo, el uso del error se define como la acción de la profesora que, a partir de una equivocación o una respuesta errónea, ayuda al alumno a que descubra, evalúe o genere una nueva comprensión. En el transcurso de las sesiones el uso del error aparece en 36 ocasiones.

Frecuentemente, los errores aparecen en las producciones de los niños tanto de escritura como de análisis fonémico. En una ocasión la profesora solicita a los niños que escriban con el alfabeto móvil la palabra dado, pero dos niños que trabajan juntos escribieron "daod". Ella pregunta si así se escribe o si hay que cambiar alguna letra. Los niños cambian de lugar una letra, entonces les dice que está bien y pueden borrar para corregir en su cuaderno. En otra sesión les pregunta qué palabra empieza con $\mathrm{P}$, un niño dice: "cama", la profesora voltea a ver a todo el grupo y pregunta si cama empieza con P; otro niño dice que la palabra "copa". La profesora vuelve a preguntar si copa empieza con P. De esta manera va utilizando el error para que los niños analicen, descubran por sí mismos y corrijan su trabajo.

El error se utiliza de manera constructiva. Cuando se utiliza el error de un niño para que todo el grupo aprenda, no se le pone en evidencia, no se le censura ni se le penaliza; se utiliza para ampliar la comprensión de todos, es decir, se usan los errores individuales para lograr el aprendizaje de quienes los cometieron y del grupo completo. En el siguiente segmento de interacción puede notarse el tono en que se analizan los errores. Uno de los niños dijo que la palabra perezoso tenía una A. La instructora escribe la palabra en el pizarrón y pregunta:

Facilitadora: ¿Dónde tiene la A perezoso, Darío?

Alumnos: No.

Facilitadora: ¿No tiene A?

Darío: No.

Facilitadora: Ooh, entonces nos confundimos.

En los intercambios anteriores puede notarse que la profesora acepta la respuesta errónea. Mediante la pregunta solicita que se haga un análisis fonético de la palabra, que los alumnos verifiquen si se incluye la letra "a" y cuando todos los niños, incluyendo quien había dado la respuesta equivocada, se cercioran de que la palabra no tiene la vocal en cuestión. En lugar de señalar al niño, asume el error como parte del trabajo del grupo, ella incluida.

Como parte del fomento de los procesos reflexivos, las instructoras llamaban la atención de los niños hacia los aspectos relevantes del conocimiento que buscaban que construyeran, les formulaban preguntas para que utilizaran su conocimiento previo o generaran nuevas comprensiones y utilizaban los errores para que a partir de ellos aprendieran, sin que estos se perci- 
bieran como algo negativo y sin penalizarlos; por el contrario, eran utilizados como oportunidades para ayudar a los niños a aprender o a precisar lo que ya sabían.

\section{Discusión}

En el estudio de la literacidad se ha criticado la enseñanza que enfatiza el conocimiento alfabético, el reconocimiento fonológico y la decodificación (Gee, 2009; Massaro, 2012), y se enfatiza el modelamiento de la lectura como una actividad social, pero también hay quienes argumentan que es necesario ayudar a los niños con estas habilidades básicas (Gutiérrez, 2018) y, sobre todo, la inclusión de un fuerte componente fonético en la enseñanza con niños que presentan dislexia (Shaywitz, 2003). En el trabajo que se realiza en el Centro Polanco se hace una enseñanza sistemática del conocimiento alfabético, el análisis fonético, así como de la decodificación, pero sin descuidar el sentido de lo leído. Dada las características de los aprendices, se considera una manera de intervención apropiada debido a que estos carecen de modelos lectores y de libros en casa, pero se reconoce que el uso social del lenguaje escrito fue poco trabajado.

Las intervenciones de las facilitadoras estuvieron encaminadas a desarrollar un proceso mental superior, comprensión y uso del código escrito, utilizando para ello distintas herramientas y símbolos creados por la cultura como lo señala Vigotsky (1979), a través del andamiaje que cumplió con ser ajustable a las necesidades de los niños, de acuerdo con lo que señalan Wood, Brunner y Ross (1974), pero no con las otras dos características de ser temporal y explícito.

Los niños tuvieron una participación activa todo el tiempo, lo que resulta acorde con la teoría constructivista, que señala la importancia de que los niños actúen sobre el objeto de conocimiento a fin de aprehenderlo, a través de un trabajo reconstructivo por parte de los aprendices (Lerner, citado por Hernández, 2000), pero a diferencia de lo que señala el enfoque, los alumnos tuvieron pocas oportunidades de revisar su propia experiencia de aprendizaje.

Frecuentemente, el trabajo se realizaba en lo que Vigotsky (1979) denominó zona del desarrollo próximo, aunque hizo falta establecer diferentes escenarios de aprendizaje que permitieran que algunos niños practicaran lo aprendido mientras que otros continuaran construyendo su conocimiento junto con las facilitadoras, que en este caso eran quienes podían más fácilmente ubicarse entre el aprendiz y el objeto de aprendizaje con el fin de ayudar a su reconstrucción.

Haywood, Brooks y Burns (1991) señalan, entre otros elementos de la mediación, ayudar a los niños a entender el sentido de lo que aprenden y a que se sientan competentes para realizar la tarea. En el presente caso, las instructoras frecuentemente resaltaban los aprendizajes de los niños, con lo que estos podían sentirse competentes; sin embargo, hubo pocas ocasiones en que se informara el sentido de la actividad.

\section{Conclusiones}

En este artículo se ha caracterizado la mediación ocurrida durante las sesiones de lectoescritura. Tales acciones se agruparon en tres grandes categorías: el sentido de la actividad y la media- 
ción, la enseñanza directa y el fomento de los procesos reflexivos. En la primera categoría, sería deseable que se fomentara más el sentido de la actividad, a fin de que los aprendices sepan qué es lo que se busca y no solamente participen en lo que se les indica.

No todas las acciones mediacionales tuvieron la misma frecuencia. Se mostró que existe una gran variabilidad. Seguramente algunas de ellas deberían de ser enfatizadas dada su relevancia. Por ejemplo, en pocas ocasiones se les comunicaba el sentido de la actividad. Esto es muy importante para que los niños busquen aprender y no solo participar, y también para que gradualmente se vuelvan aprendices autónomos.

A pesar de que las acciones que se describieron eran adecuadas para que los aprendices desarrollaran algunas habilidades fonológicas, reconocieran letras y escribieran palabras, solo se vio un avance modesto en el dominio de las habilidades básicas para escribir y leer, probablemente debido a la falta de un seguimiento más preciso del aprendizaje de cada alumno, pues aunque las interacciones ocurrían en la zona del desarrollo próximo de algunos de los niños, se pasaban rápidamente al trabajo con otro, pero no se aseguraban de que cada uno de ellos fuera internalizando lo aprendido.

Los aprendices fueron niños que no habían logrado aprender a leer y escribir en la escuela con los métodos convencionales, provenían de hogares con escasa o nula práctica lectora y tuvieron una asistencia variable. Esos elementos ayudarían a explicar parcialmente la razón del mediano desarrollo de la competencia de literacidad, pero a partir de los datos analizados, se puede formular la hipótesis de que hubo una falla importante en la enseñanza, pues a pesar de que las lecciones estaban planeadas, que había un trato cálido y respetuoso hacia los niños y que se utilizaban múltiples acciones mediacionales, no se construyeron escenarios diferenciados (asesorías individuales, trabajo individual, etc.) que permitieran a algunos niños practicar lo recién aprendido, mientras que otros continuaran reconstruyendo conocimientos en su zona del desarrollo próximo con la ayuda de las profesoras.

Se notó que los niños que ya dominaban algún conocimiento tenían que seguir participando con todo el grupo en actividades que ya no les aportaban y, cuando se atendían asuntos más complejos, estos quedaban fuera del alcance de quienes tenían un menor desarrollo.

Las acciones mediacionales utilizadas por las facilitadoras producían efecto inmediato en el aprendizaje de los niños, por lo que se considera que convendría continuar con el trabajo planeado, sistemático y con la atención respetuosa y cálida a los aprendices. La investigación aporta una descripción de buenas prácticas de mediación y atención a niños que enfrentan dificultades para el desarrollo de la competencia de literacidad, así como recomendaciones que, aunadas a las buenas prácticas, podrían incidir en un aprendizaje más rápido y eficaz: asegurarse de que los niños comprendan el sentido de lo que hacen, que el conocimiento se utilice con fines sociales $y$, sobre todo, que se haga una enseñanza diferenciada para que se trabaje y dé seguimiento en la zona del desarrollo próximo de cada niño, para que se potencie el impacto de la enseñanza en el nivel de desempeño de los aprendices del código escrito. 


\section{Referencias}

Asociación Americana de Psiquiatría (2014). Manual diagnóstico y estadístico de los trastornos mentales. Madrid: Editorial Médica Panamericana.

Baker, D. L.; R. H. Good; N. Knutson; J. M. Watson (eds.). (2006). Indicadores dinámicos del éxito en la lectura (7a ed.). Eugene: University of Oregon, Dynamic Measurement Group. http:// dibels.uoregon.edu

Baquero, R. (1996). Vygotsky y el aprendizaje escolar. Buenos Aires: Aique.

Cassany, D. (2005). Investigaciones y propuestas sobre literacidad actual: multiliteracidad, internet y criticidad. Congreso Nacional Cátedra Unesco para la Lectura y la Escritura. Concepción: Universidad de Concepción. http://www2.udec.cl/catedraunesco/05CASSANY.pdf

Cassany, D. (2006). Tras las líneas. Sobre lectura contemporánea. Barcelona: Anagrama.

Feuerstein, R.; Y. Rand; M. B. Hoffman; R. Miller (1980). Instrumental Enrichment: An Intervention Program for Cognitive Modifiability. Baltimore: University Park Press.

Fletcher, J. M.; D. J. Francis; R. D. Morris; G. R. Lyon (2005). Evidence-based Assessment of Learning Disabilities in Children and Adolescents. Journal of Clinical Child and Adolescent Psychology, 34(3), 506-522. https://doi.org/10.1207/s15374424jccp3403 7

Gee, J. P. (2009). Situated Language and Learning: A Critique of Traditional Schooling. Londres: Routledge.

Gómez-Palacio, M.; M. Cárdenas; E. Guajardo; A. Kaufman; M. Maldonado; N. Richero; I. Velázquez (1984). Propuestas para el aprendizaje de la lengua escrita. México: SEP-OEA.

Gutiérrez, R.; A. Díez (2017). Conciencia fonológica, desarrollo evolutivo de la escritura en las primeras edades. Educación XX1, 21(1).

Gutiérrez, R. (2018). Habilidades favorecedoras del aprendizaje de la lectura en alumnos de 5 y 6 años. Revista Signos, 51(96), 45-60.

Haywood, H.; Brooks, P.; Burns, S. (1991). Cognitive Curriculum for Young Children. En A. Costa (ed.). Developing Minds Programs for Teaching Thinking. Virginia: Association for Supervision and Curriculum Development, 107-109.

Hernández, G. (2000). Paradigmas en psicología de la educación. México: Paidós.

INEE (2007). El aprendizaje en tercero de primaria en México: español, matemáticas, ciencias sociales y ciencias naturales. México: INEE.

INEE (2019). Panorama educativo de México. Indicadores del Sistema Educativo Nacional 2018. Educación básica y media superior. México: INEE. https://www.inee.edu.mx/wp-content/uploads/2019/08/P1B117.pdf

Massaro, D. W. (2012). Acquiring Literacy Naturally. American Scientist, 100(4), 324-333. https:// doi-org.ezproxy.iteso.mx/10.1511/2012.97.324

Miles, M.; M. Huberman (1994). Qualitative Data Analysis: An Expanded Sourcebook. Thousand Oaks: Sage Publications. 
Moura, O.; M. Pereira; J. Moreno; M. Simões (2020). Investigating the Double-deficit Hypothesis of Developmental Dyslexia in an Orthography of Intermediate Depth. Annals of Dyslexia (70), 43-61. https://doi.org/10.1007/s11881-020-00190-1

Neiman, G.; G. Quaranta (2012). Los estudios de caso en la investigación cualitativa. En Vasilachis, I. (coord.). Estrategias de investigación cualitativa. Barcelona: Gedisa.

OCDE (2018). PISA for Development Assessment and Analytical Framework: Reading, Mathematics and Science. París: OECD Publishing. http://dx.doi.org/10.1787/9789264305274-en

OCDE (2019). PISA 2018 Results (Volume l): What Students Know and Can Do. París: PISA, OECD Publishing. https://doi.org/10.1787/5f07c754-en

Shaywitz, R. (2003). Overcaming Dislexia: A New and Complete Science Based Program for Reading Problems at any Level. Nueva York: A. A. Knopf.

Stake, R. (1999). Investigación con estudio de casos. Madrid: Morata.

Tamayo, S. (2017). La dislexia y las dificultades en la adquisición de la lectoescritura. Profesorado. Revista de Currículum y Formación de Profesorado, 21(1),423-432.

https://www.redalyc.org/articulo.oa?id=567/56750681021

UNESCO (2017). Factsheet No. 45. http://unesdoc.unesco.org/images/0025/002589/258942e.pdf Vigotsky, L. (1979). El desarrollo de los procesos psicológicos superiores. Barcelona: Grijalbo.

Wood, D.; J. S. Bruner; G. Ross (1976). The Role of Tutoring in Problem Solving. Journal of Child Psychology and Psychiatry, 17(2), 89-100. 